\title{
Sex differences and factors influencing the duration of the QT interval in patients on anti-tuberculosis therapy
}

To the Editor:

In an ECG, the QT interval represents the depolarisation and repolarisation of the ventricles and is usually corrected for heart rate (QTc). Previous studies have shown that women have a reduced repolarisation reserve and greater susceptibility to the QTc prolongation effect of some drugs $[1,2]$.

Bedaquiline and delamanid prolong the QTc and present a challenge when treating multidrug-resistant tuberculosis (TB) [3, 4]. However, little is known about the QTc effects of standard first-line drugs, because initial studies for these drugs were performed before it became customary to assess QTc prolongation effects in advance of introducing a new medicine to the market [3]. In this study, we aimed to find factors influencing the duration of the QTc in patients with TB and, in particular, the influence of sex on the QTc prolongation effect of TB drugs.

For the preparation of this article, we used data from the OFLOTUB trial, which compared a standard 6-month treatment, including rifampicin $(\mathrm{R})$, isoniazid $(\mathrm{H})$, pyrazinamide $(\mathrm{Z})$ and ethambutol $(\mathrm{E})$, with a 4-month treatment containing gatifloxacin $(G)$ [5]. Patients with bradycardia or long QTc at baseline, taking drugs known to prolong the QTc, on antiretroviral therapy, or with hypokalaemia were excluded. During the first 2 months of treatment, patients received RHZE in the standard treatment arm and RHZG in the gatifloxacin arm. In the continuation phase, patients received RH in the standard treatment arm and RHG in the gatifloxacin arm. ECGs were performed before treatment initiation and $1-5 \mathrm{~h}$ post-dosing of TB drugs at week 4, week 8 and the end of treatment. A Schiller CP300 electrocardiograph (Schiller Healthcare India Pvt. Ltd., Mumbai, India) was used to automatically calculate QT intervals and heart rate at all sites and heart rate correction was performed using the Fridericia formula [6]. Full details of the study can be obtained elsewhere [5].

For the mathematical longitudinal modelling, we included variables known to be risk factors for QTc prolongation (age, sex, HIV co-infection, body mass index (BMI), mean arterial blood pressure (MAP), temperature and serum potassium concentration) [7, 8]. We constructed a mixed-effect linear regression model with random intercepts [9]. In a sensitivity analysis, we also included a fixed-effect linear regression model, which only took into account the variability within subjects, and therefore estimations were not affected by confounding from unmeasured time-invariant factors [10]. We assessed sex differences by introducing an interaction term between TB regimens and sex. To interpret the interaction, estimations of the effect of TB drugs on the QTc were segregated by sex.

The multivariate multilevel mixed-effect model included 5889 observations from 1660 patients with an average of 3.5 observations per patients (range 1-5). Variables associated with a significant prolongation of the QTc were female sex $(2.98 \mathrm{~ms}, 95 \%$ CI $0.52-5.43)$, age $(0.27 \mathrm{~ms}$ per year, $95 \%$ CI $0.18-0.35)$, HIV co-infection (2.25 ms, 95\% CI 0.19-4.31) and BMI (0.49 ms per BMI unit in $\mathrm{kg} \cdot \mathrm{m}^{-2}, 95 \%$ CI $\left.0.20-0.77\right)$. Significant shortening of the QTc was observed with increases in temperature $\left(-2.75 \mathrm{~ms}\right.$ per ${ }^{\circ} \mathrm{C}, 95 \%$ CI $-3.53--1.97)$ and serum potassium concentration $\left(-0.96 \mathrm{~ms}\right.$ per $\left.\mathrm{mmol} \cdot \mathrm{L}^{-1}, 95 \% \mathrm{CI}-1.88--0.05\right)$. MAP variation was not associated with a significant change in the QTc $(0.01 \mathrm{~ms}$ per $\mathrm{mmHg}, 95 \% \mathrm{CI}-0.05-0.08)$. All interaction terms between female sex and each one of the TB regimens (RH, RHG, RHZE and RHZG) were statistically significant ( $p$-value $<0.0001$ ). The effect on the QTc of each TB regimen segregated by sex is presented in figure 1. Reassuringly, the mixed-effects and the fixed-effect models gave similar results. All

@ERSpublications

Sex has a strong influence on the QTc prolongation effect of first-line TB drugs and gatifloxacin http://ow.ly/UXtP30hJJCu

Cite this article as: Alvarez-Uria G, Midde M. Sex differences and factors influencing the duration of the QT interval in patients on anti-tuberculosis therapy. Eur Respir J 2018; 51: 1702368 [https://doi.org/ 10.1183/13993003.02368-2017]. 

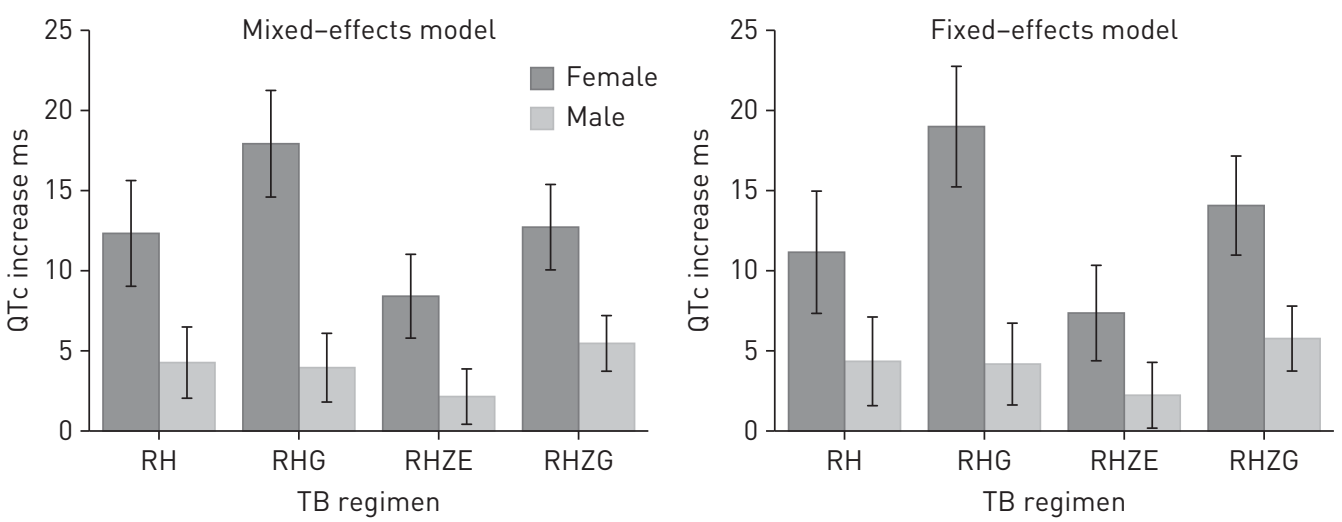

FIGURE 1 Increase in the duration of the corrected QT (QTC) interval after initiation of anti-tuberculosis (TB) therapy. R: rifampicin; H: isoniazid; G: gatifloxacin; Z: pyrazinamide; E: ethambutol. Error bars represent $95 \%$ Cls for the estimation.

TB regimens increased the duration of the QTc, but the magnitude of the effect was larger in female patients. In the mixed-effect model, the use of RH, RHG, RHZE and RHZG increased the duration of the QTc by $12.33 \mathrm{~ms}$ (95\% CI 9.03-15.63), $17.92 \mathrm{~ms}$ (95\% CI 14.6-21.25), $8.41 \mathrm{~ms}$ (95\% CI 5.8-11.02) and $12.72 \mathrm{~ms}$ (95\% CI 10.05-15.38) in women, and by $4.27 \mathrm{~ms}$ (95\% CI $2.05-6.49$ ), $3.95 \mathrm{~ms}$ (95\% CI $1.81-$ 6.09), $2.15 \mathrm{~ms}$ (95\% CI 0.42-3.87) and $5.46 \mathrm{~ms}$ (95\% CI 3.73-7.2) in men, respectively. Because sex differences could be explained by the fact that the mean weight was lower in women, and hence women could have been overexposed to TB drugs, we performed a sensitivity analysis by adjusting the results by weight instead of BMI: this showed almost identical results (data not shown). When comparing regimens with and without gatifloxacin (RHG or RHZG versus RH or RHZE), the use of gatifloxacin was associated with a significant increase in the QTc in women $(7.27 \mathrm{~ms}, 95 \%$ CI 3.23-11.32) but not in men (1.68 ms, 95\% CI -0.81-4.18). These results are in agreement with a review of 14 crossover studies of the effect of moxifloxacin on QTc, which showed that women exhibited larger mean effect and variabilities than men [11]. Fluoroquinolones are one of the most important drugs in the treatment of multidrug-resistant TB and are frequently combined with other drugs that prolong the QTc, such as clofazimine, delamanid or bedaquiline [3].

None of the first-line drugs used in the standard TB regimen is usually included in the list of drugs that prolong the QTc [7]. While the magnitude of the effect can seem relatively small, the mathematical models estimated average effects in a selected group of patients from a clinical trial with extensive exclusion criteria and close monitoring. In 2016 there were 10.4 million cases of TB, 35\% in women [12]. Even small average increases in the QTc may translate to a substantial number of patients developing drug-induced long QTc syndrome when treating a large population [3]. TB programmes and clinicians treating "real-life" pulmonary TB should be aware of the QTc prolongation effect of standard first-line TB regimens, especially in women or when other risk factors for long QTc syndrome are present.

The study has some limitations. The QTc was measured automatically by an electrocardiograph with no external quality controls by human readings. Although automatic algorithms can improve the consistency and reproducibility of the measurements, they may lead to inaccurate results in the presence of noise, abnormal rhythms, low amplitude T waves or overlapping U waves [13]. Moreover, the OFLOTUB study was performed in an African population, and previous reports have shown substantial heterogeneity in the duration of the QTc among ethnic groups [14]. New studies are needed to confirm our findings in other populations.

In conclusion, female sex, HIV co-infection, older age and a higher BMI were associated with QTc prolongation, while increases in temperature and serum potassium concentrations shortened the QTc. Clinicians and TB programmes should be aware that patients starting standard first-line TB regimens could experience a mild prolongation of the QTc and could be at risk of long QTc syndrome, especially women. Our findings support the routine evaluation of sex differences when studying the QTc prolongation effect of TB drugs.

Gerardo Alvarez-Uria (i) and Manoranjan Midde

Dept of Infectious Diseases, Rural Development Trust Hospital, Bathalapalli, India.

Correspondence: Gerardo Alvarez-Uria, Dept of Infectious Diseases, Bathalapalli Rural Development Trust Hospital, Kadiri Road, Bathalapalli 515661, Anantapur District, Andhra Pradesh, India. E-mail gerardouria@gmail.com 
Received: Nov 142017 | Accepted after revision: Dec 202017

Conflict of interest: None declared.

Acknowledgements: Data used in the preparation of this article were obtained from the Platform for Aggregations of Clinical TB Studies (codr.c-path.org). The Platform for Aggregations of Clinical TB Studies initiative is a public-private partnership launched in May 2015 by Critical Path Institute (C-Path), the Special Programme for Research and Training in Tropical Diseases (TDR), the Global Alliance for TB Drug Development (TB Alliance), and St. George's University of London.

\section{References}

1 Shah RR, Morganroth J. ICH E14 Q \& A (R1) document: perspectives on the updated recommendations on thorough QT studies. Br J Clin Pharmacol 2013; 75: 959-965.

2 Li G, Cheng G, Wu J, et al. Drug-induced long QT syndrome in women. Adv Ther 2013; 30: 793-802.

3 Harausz E, Cox H, Rich M, et al. QTc prolongation and treatment of multidrug-resistant tuberculosis. Int J Tuberc Lung Dis 2015; 19: 385-391.

4 Pontali E, Sotgiu G, Tiberi S, et al. Cardiac safety of bedaquiline: a systematic and critical analysis of the evidence. Eur Respir J 2017; 50: 1701462.

5 Merle CS, Fielding K, Sow OB, et al. A four-month gatifloxacin-containing regimen for treating tuberculosis. N Engl J Med 2014; 371: 1588-1598.

6 Olliaro PL, Merle C, Mthiyane T, et al. Effects on the QT interval of a gatifloxacin-containing regimen versus standard treatment of pulmonary tuberculosis. Antimicrob Agents Chemother 2017; 61: 01834-16.

7 Vandael E, Vandenberk B, Vandenberghe J, et al. Risk factors for QTc-prolongation: systematic review of the evidence. Int J Clin Pharm 2017; 39: 16-25.

8 Wongcharoen W, Suaklin S, Tantisirivit N, et al. QT dispersion in HIV-infected patients receiving combined antiretroviral therapy. Ann Noninvasive Electrocardiol 2014; 19: 561-566.

9 Rabe-Hesketh S, Skrondal A. Multilevel and longitudinal modeling using Stata. 3rd Edn. College Station, TX, Stata Press Publication, 2012.

10 Gunasekara FI, Richardson K, Carter K, et al. Fixed effects analysis of repeated measures data. Int J Epidemiol 2014; 43: 264-269.

11 Yan LK, Zhang J, Ng MJ, et al. Statistical characteristics of moxifloxacin-induced QTc effect. J Biopharm Stat 2010; 20: 497-507.

12 World Health Organization. Global tuberculosis report 2017. Geneva, WHO, 2017.

13 Food and Drug Administration. E14 clinical evaluation of QT/QTc interval prolongation and proarrhythmic potential for non-antiarrhythmic drugs. Questions and answers (R3) guidance for industry. www.fda.gov/ downloads/drugs/guidancecomplianceregulatoryinformation/guidances/ucm073161.pdf Date last accessed: August $8,2017$.

14 Shah RR. Drug-induced QT interval prolongation: does ethnicity of the thorough QT study population matter? $\mathrm{Br}$ J Clin Pharmacol 2013; 75: 347-358. 